Isolation and Characterization of Residual Undifferentiated Mouse Embryonic Stem Cells from Embryoid Body Cultures by Fluorescence Tracking Author(s): Roberto Enseñat-Waser, Alfredo Santana, Néstor Vicente-Salar, Juan C. Cigudosa, Enrique Roche, Bernat Soria and Juan A. Reig Source: In Vitro Cellular \& Developmental Biology. Animal, Vol. 42, No. 5/6 (May - Jun., 2006), pp. 115-123

Published by: Society for In Vitro Biology

Stable URL: https://www.jstor.org/stable/4295680

Accessed: 06-05-2021 14:54 UTC

JSTOR is a not-for-profit service that helps scholars, researchers, and students discover, use, and build upon a wide range of content in a trusted digital archive. We use information technology and tools to increase productivity and facilitate new forms of scholarship. For more information about JSTOR, please contact support@jstor.org.

Your use of the JSTOR archive indicates your acceptance of the Terms \& Conditions of Use, available at https://about.jstor.org/terms

Society for In Vitro Biology is collaborating with JSTOR to digitize, preserve and extend access to In Vitro Cellular \& Developmental Biology. Animal 


\title{
ISOLATION AND CHARACTERIZATION OF RESIDUAL UNDIFFERENTIATED MOUSE EMBRYONIC STEM CELLS FROM EMBRYOID BODY CULTURES BY FLUORESCENCE TRACKING
}

\author{
ROBERTO ENSEÑAT-WASER, ALFREDO SANTANA, NÉSTOR VICENTE-SALAR, JUAN C. CIGUDOSA, ENRIQUE ROCHE, \\ BERNAT SORIA, AND JUAN A. REIG
}

\begin{abstract}
Instituto de Bioingeniería, Unidad de Bioquímica y Terapia Celular, Universidad Miguel Hernández, Elche, Alicante, Spain (R. E.-W. A. S., N. V.-S., E. R., B. S., J. A. R), Centro Nacional Investigaciones Oncológicas, Madrid, Spain (J. C. C.)
\end{abstract}

(Received 27 September 2005; accepted 6 January 2006)

\begin{abstract}
SUMmaRY
The differentiation of mouse embryonic stem (ES) cells can be induced in vitro after leukemia inhibitory factor (LIF) withdrawal and further enhanced by the formation of "embryoid body" (EB) aggregates. This strategy is being used in order to optimize differentiation protocols that would result in functional cells for experimental cell replacement therapies. However, this study presents the possibility for residual undifferentiated cells to survive after standard in vitro procedures. Mouse ES cells were stably transfected with the enhanced green fluorescent protein (EGFP), under the control of the Oct 4 promoter, a transcription factor that is expressed in undifferentiated ES cells but down-regulated on differentiation. Residual fluorescent cells were isolated from EBs that were cultured in standard conditions in absence of LIF. These residual cells displayed recurrent gain of chromosomes 8 and 9. Residual fluorescent cells, further expanded in absence of LIF and cultured as EBs, still displayed a significant Oct4 expression in comparison with parental transfected ES cells. Consequently, these residual cells have an intrinsic resistance to differentiate. The behavior of these cells, observed in vitro, can be overcome in vivo, as they were able to induce teratomas in subcutaneously injected nude mice. Residual undifferentiated cells displayed slight levels of VASA and DAZL expression. These results demonstrate that mouse ES cells cultured in vitro, in standard conditions, can spontaneously acquire recurrent karyotypical changes that may promote an undifferentiated stage, being selected in standard culture conditions in vitro.
\end{abstract}

Key words: cell differentiation; cell culture; tumorigenicity; flow cytometry; Oct4, karyotype.

\section{INTRODUCTION}

Different mouse embryonic stem (ES) cell lines, derived from preimplantation embryos, are being used to study the differentiation process of embryonic pluripotent cells in vitro (Nagy et al., 1993). In the presence of leukemia inhibitory factor (LIF), these cells can proliferate indefinitely in an undifferentiated state because of the cytokine-mediated activation of the transcription factor STAT3 (signal transducer and activator of transcription) (Raz et al., 1999) and the maintenance of Oct4 expression, a POU-domain transcription factor (Nichols et al., 1998). Nevertheless, the relationship of these two pathways is not well known. Oct4 is one of the initial transcription factors expressed in mammalian embryogenesis. It is characterized by its exclusive expression in blastomeres, in the inner cell mass of the blastocyst, and in the germ cell lineage (Rosner et al., 1990). Oct4 expression is down-regulated during gastrulation, when cells differentiate into the three germ layers, and there is no apparent expression in somatic lineages. Different pluripotent stem cells express Oct4 in culture, including ES cells, embryonal carcinoma cells, and primordial germ cells (PGCs). Therefore, Oct4 is a key marker of cells with pluripotency, both in vivo and in vitro

${ }^{1}$ To whom correspondence should be addressed at Instituto de Bioingeniería, Universidad Miguel Hernández, Alicante 03550, Spain. E-mail: jareig@umh.es
(Niwa et al., 2001). However, Oct4 not only acts on the control of pluripotency but also regulates differentiation programs in embryonic cells, according to its level of expression as well as other conditions that have not yet been completely established (Niwa et al., 2000). Consequently, Oct4 null embryos cannot develop properly since the derived blastocysts are nonviable (Nichols et al., 1998). ES cells spontaneously differentiate in vitro after LIF withdrawal in multicellular aggregates called embryoid bodies (EBs) when cultured in suspension (Doetschman et al., 1985). In these standard culture conditions, Oct4 is down-regulated in a few days, and the differentiation process continues (Buehr et al., 2003). During the normal course of embryogenesis, pluripotent cells, in the inner cell mass of the blastocyst, give rise to the primitive endoderm as well as the primitive ectoderm, which originates the three germ layer lineages, as well as the germ cells and additional extraembryonic tissues (Gardner et al., 1979). The developmental potency of EBs has also been studied in vitro using different pluripotent ES cell lines (Prelle et al., 1999). In these conditions, the differentiation program closely resembles early mouse embryogenesis, which includes the gradual expression of the primitive endoderm, primitive ectoderm, and derivative lineages (Murray et al., 2001). Differentiation agents such as retinoic acid, as well as conditioned or defined media, have subsequently been used in the outgrow phase to further enhance in vitro differentiation with several differences in 
the timing or the extension of the different cell lineages (Hopfl et al., 2004). The possible derivation and culture of human ES cells (Thomson et al., 1998) has increased the interest in the study of in vitro differentiation as well as the development of strategies that may allow specific cell types to be obtained. Several approaches have been used to obtain functional cells by directing the differentiation process, preferentially toward one single cell fate. Gene trapping (Roche et al., 2003) or the manipulation of the culture media has been used for the isolation of different cell types, including cardiomyocytes (Klug et al., 1996), neurons (Kim et al., 2002), and insulin-producing cells (Soria et al., 2000), which have been used in order to restore an impaired cellular function in animal models.

A genetic construction containing the Oct 4 gene promoter, regulating the expression of the enhanced green fluorescent protein (EGFP), has been utilized in this research in order to study the differentiation progress of transfected mouse ES cells. We have characterized a remnant population of fluorescent cells from EBs, after a standard culture period, which does not follow the established differentiation program. This population could be heterogeneous, containing also previously characterized PGCs in an early stage of differentiation (Toyooka et al., 2003; Geijsen et al., 2004), according to the slight expression of VASA and DAZL. When these residual cells were isolated by cell sorting and further grown in absence of LIF, they showed a significant expression of Oct4, even after EB formation, as opposed to parental transfected R1 ES cells. Additionally, residual cells were not able to differentiate in vitro after EBs formation. When these cells were subcutaneously injected into immunodeficient mice, they induced teratomas, suggesting that they possess a limited developmental potency in vitro, yet at the same time they can be reactivated in vivo by signals that are still unknown. Karyotypical alterations observed in these residual cells suggest that they could spontaneously acquire a selective advantage during prolonged cultures supporting a prevalent undifferentiated condition in vitro.

\section{Materials AND Methods}

ES-cell culture. We have used the ES cell line R1 (passages 20-30) (Nagy et al., 1993). For undifferentiated culture, cells were grown on gelatin-coated $(0.1 \%$ in phosphate-buffered saline [PBS]; Sigma Chemical Co., St. Louis, MO) dishes in Dulbecco's modified Eagle medium (DMEM; high glucose) supplemented with $15 \%$ heat-inactivated fetal bovine serum (FBS; selected batches; Biochrom, Berlin, Germany), $2 \mathrm{~m} M$ L-glutamine, $0.1 \mathrm{~m} M \beta$-mercaptoethanol, nonessential amino acids (stock solution $100 \times$ ), $100 \mathrm{U} / \mathrm{ml}$ penicillin, $100 \mu \mathrm{g} / \mathrm{ml}$ streptomycin, and $1000 \mathrm{U} / \mathrm{ml}$ recombinant murine leukemia inhibitory factor (mLIF; ESGRO ${ }^{\circledR}$, Chemicon). Other cell culture reagents were purchased from Invitrogen (Carlsbad, CA). The EBs were generated by aggregation of ES cells, transferring $2-5 \times 10^{5}$ cells $/ \mathrm{ml}$ to bacteriological Petri dishes in the previously mentioned culture medium, where cell differentiation was induced by excluding mLIF from medium and reducing FBS to $10 \%$. The course of EB differentiation was studied at different periods of time, and they were maintained in culture for up to $30 \mathrm{~d}$.

Plasmid construction and cell transfection. The 3.19-kb Oct4 promoter was ligated to the 0.74-kb EGFP coding sequence attached to a BGH polyA sequence. In order to allow the selection of transfected cells, the construct also included a pGK-hygro-pA sequence, which allows constitutive expression of hygromicin resistance. Whole construct was $9.1 \mathrm{~kb} ; 3 \times 10^{7}$ cells were electroporated with $60 \mu \mathrm{g}$ of XbaI (Roche, Bazel, Switzerland) linearized construct using a Gene Pulser II ${ }^{\circledR}$ (BioRad, Hercules, CA), applying one pulse of $0.8 \mathrm{kV}$ and $3 \mu \mathrm{F}$. Cell suspension was then plated at a density of $3 \times 10^{6}$ cells/dish onto 100 -mm gelatinized culture dishes. Selection of transfected cells was initiated after $2 \mathrm{~d}$ by the addition of $200 \mu \mathrm{g} / \mathrm{ml}$ hygromicin (Invitrogen) to the media.
Pluripotency assays by alkaline phosphatase and SSEA-1 staining. Alkaline phosphatase expression was determined by staining with the Vector Red ${ }^{\circledR}$ Alkaline Phosphatase substrate Kit I (Vector Laboratories, Burlingame, CA). The Solter and Knowles method (Solter et al., 1978) was used for the SSEA1 (stage-specific embryonic antigen-1) immunodetection. Primary antibodies against SSEA-1 (MC-480) were obtained from the Developmental Studies Hybridoma Bank (University of Iowa, Ames, IA). Secondary antibodies against mouse IgM conjugated with TRITC were used at a concentration of 1:125 (Jackson Immunoresearch, West Grove, PA). Images were taken with an Eclipse TE200 microscope (Nikon).

Reverse transcription/polymerase chain reaction. The EBs were collected on different days and washed twice with PBS, and total RNA was isolated using Trizol ${ }^{\circledR}$ Reagent (Invitrogen). One microgram of RNA was reverse transcribed using Expand Reverse Transcriptase and oligo-dT primers (Roche), following manufacturer recommendations. Aliquots of $0.5 \mu \mathrm{l}$ of the reverse transcription products were used for the polymerase chain reaction using specific primers and the Expand High Fidelity PCR System (Roche). The mixture was denatured for 5 min at $95^{\circ} \mathrm{C}$, followed by 26 cycles of 15 -s denaturing at $94^{\circ} \mathrm{C}, 35 \mathrm{~s}$ at the corresponding annealing temperature, and $1 \mathrm{~min} 30 \mathrm{~s}$ elongation at $72^{\circ} \mathrm{C}$, plus a final elongation of $10 \mathrm{~min}$ at $72^{\circ} \mathrm{C}$. Primer sequences, annealing temperatures, and amplified fragment sizes are (antisense followed by sense) as follows:

OCT4:

5'-AGGCCCGGAAGAGAAAGCGAACTA-3'

$5^{\prime}$-TGGGGGCAGAGGAAAGGATACAGC-3'; $67^{\circ} \mathrm{C}, 265$ base pairs (bp)

$\alpha$-Fetoprotein:

5'-CCTTGGCTGCTCAGTACGACAAGG-3'

$5^{\prime}$-CCTGCAGACACTCCAGCGAGTTTC- $3^{\prime} ; 67^{\circ} \mathrm{C}, 301 \mathrm{bp}$

Neurofilament 200:

5'-GAGTGGTTCCGAGTGAGGTTGGAC-3'

5'-GACGTTGAGCAGGTCCTGGTACTC-3'; $67^{\circ} \mathrm{C}, 302 \mathrm{bp}$

$\beta$-MHC:

5'-GCCAACACCAACCTGTCCAAGTTC-3

5'-TGCAAAGGCTCCAGGTCTGAGGGC-3'; 64 C, 205 bp

Nanog:

5'-AGGGTCTGCTACTGAGATGCTCTG-3'

$5^{\prime}$-CAACCACTGGTTTTTCTGCCACCG-3'; $62^{\circ} \mathrm{C}, 364 \mathrm{bp}$

ESG-1:

5'-ATAAGCTTGATCTCGTCTTCC-3'

5'-CTTGCTAGGATGTAACAAAGC-3'; 55 C, 501 bp

DAZL:

5'-GCCAGCACTCAGTCTTCATC-3'

5'-GTTGGAGGCTGCATGTAAGT-3'; 57 $\mathrm{C}, 419 \mathrm{bp}$

VASA:

5'-GGCAAAGAAAAGATTGGCCT-3'

5'-5'-GGGTTTGGCGTTGTTCCTT- $3^{\prime} ; 62^{\circ}$ C, 141 bp

Actin was used as a housekeeping control gene:

5'-AGAGGGAAATCGTGCGTGAC-3'

5'-CAATAGTGATGACCTGGCCGT-3' $60^{\circ} \mathrm{C}, 258 \mathrm{bp}$

Amplification products were analyzed by electrophoresis in $2 \%$ agarose gels.

Oct4 expression was assessed in some experiments by semiquantitative reverse transcription/polymerase chain reaction (RT-PCR). In these cases, $1 \mu l$ of the reverse transcription products were used for PCR reactions. Initially the linear phase of amplification was determined, and PCR was subsequently performed in triplicates using 25 cycles and the following primers, annealing temperatures and amplified fragment sizes (antisense followed by sense):

OCT4:

5'-TTCTGGCGCCGGTTACAGAACCA-3

5'-GACAACAATGAGAACCTTCAGGAGA-3'; 60 $\mathrm{C}, 217 \mathrm{bp}$

$\beta$-Actin:

5'-TCCCAGTTGGTAACAATGCCA-3'

$5^{\prime}$-CCCTAGGCACCAGGGTGTGA-3'; $60^{\circ} \mathrm{C}, 128 \mathrm{bp}$

PCR products were visualized on agarose gels, subjected to densitometric scanning (Quantity One Software, BioRad), and standardized against $\beta$-actin in parallel reactions.

Real-time PCR. The cDNA was synthesized as described previously; 0.25 $\mu l$ of those products was used for a quantitative PCR reaction using the adequate primers and the LightCycler FastStart DNA MasterPLUS SYBR Green I (Roche). Triplicate reactions for each sample were performed in a 

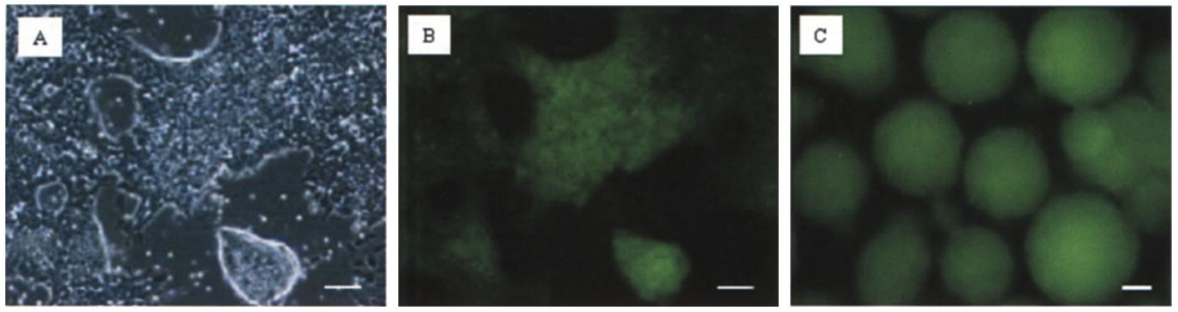

FIG. 1. Selection of Rl mouse embryonic stem (ES) cells transfected with the Oct4EGFP construct. (A) Phase contrast and $(B)$ green fluorescence of representative clones selected in presence of hygromycin after $48 \mathrm{~h}$ in monolayer culture. $(C)$ Green fluorescence of embryoid bodies (EBs) originated from Oct4EGFP transfected cells after $24 \mathrm{~h}$ in suspension culture. Bar, $100 \mu \mathrm{m}$. EGFP, enhanced green fluorescent protein.

Light Cycler, real-time thermocycler (Roche). Absolute quantification was achieved after extrapolation of the calculated $\Delta \mathrm{Ct}$ in the corresponding standard curve. The number of copies for each gene was normalized to 10,000 copies of actin. The PCR program consisted of an initial step at $95^{\circ} \mathrm{C}$ for $10 \mathrm{~min}$ followed by 40 cycles of 10 -s denaturing at $95^{\circ} \mathrm{C}, 7 \mathrm{~s}$ at $62^{\circ} \mathrm{C}$, and $12 \mathrm{~s}$ at $72^{\circ} \mathrm{C}$, plus a final melting curve step. Primer sequences were as follows:

Oct-4:

Fw 5'-GGCGTTCTCTTTGGAAAGGT-3', Rv 5'-TCTCATTGTTGTCGGCTT

CCT-3'

Mvh (vasa):

Fw 5'-GGCAAAGAAAAGATTGGCCT-3', Rv 5'-GGGTTTGGCGTTGTTCC TT- $3^{\prime}$, and $\beta$-actin used as housekeeping gene

Fw 5'-CCCTAGGCACCAGGGTGTGA-3', Rv 5'-TCCCAGTTGGTAACAATGCCA-3'

FACS analysis. Cells were resuspended at a final concentration of $10^{6}$ cells/ $\mathrm{ml}$, in PBS, containing $1 \mathrm{~m} M$ EDTA. A FACS Vantage SE System (BD Biosciences, San José, CA) was used to assess the fluorescence and cell counts for each intensity. This instrument was also used to isolate EGFP-positive cells from EBs cultured $20 \mathrm{~d}$. Total event rate did not exceed 1000 cells per second. The efficiency and purity of isolated cells was determined using fluorescence microscopy. To obtain homogeneous cell suspensions, both plated cells and EBs were either trypsinized or, in the case of dense cell aggregates, digested with $250 \mathrm{U} / \mathrm{ml}$ of collagenase (Sigma) in PBS with $1 \mathrm{~m} M$ EDTA for $50 \mathrm{~min}$ at $37^{\circ} \mathrm{C}$ and then diluted 1:1 with trypsin/EDTA $1 \times$ solution (Invitrogen) for $15 \mathrm{~min}$ at $37^{\circ} \mathrm{C}$. Cell disaggregation was achieved by pipetting through

5 -ml pipette for $5 \mathrm{~min}$. The supernatant cells were further used after residual cell clusters were allowed to settle for $5 \mathrm{~min}$. Cells were finally counted, centrifuged, and resuspended to the final concentration described previously in PBS containing $1 \mathrm{~m} M$ EDTA.

Karyotype analysis. Chromosome number was obtained by using Giemsa banding after analyzing at least 60 metaphase cells from each cell preparation. For molecular cytogenetic analysis, a spectral karyotyping (SKY) analysis was carried out as previously described (Rubio et al., 2005). Cells were exposed to colchicine $(0.5 \mu \mathrm{g} / \mathrm{ml})$ for $4 \mathrm{~h}$ at $37^{\circ} \mathrm{C}$ and harvested routinely. Metaphases were prepared from the different cell types following a conventional cytogenetic protocol for methanol-acetic acid (3:1)-fixed cells. Slides were prepared from the fixed material and hybridized using the SKY method, according to the manufacturer's protocol (Applied Spectral Imaging, Migdal $\mathrm{Ha}$ 'Emek, Israel). Images were acquired with an SD300 Spectra Cube (Applied Spectral Imaging) mounted on a Zeiss Axioplan microscope using a custom-designed optical filter, SKY-1 (Chroma Technology, Brattleboro, VT). Up to 20 metaphase cells were captured and analyzed for each cell line whenever possible.

\section{RESLLTS}

Oct4-EGFP cell selection. The R1 ES cells were stably transfected with the Oct4-EGFP construct, as described in Materials and Methods, and several clones displaying the highest levels of fluorescence were selected, expanded in monolayer (Fig. $1 A$ and $B$ ), and analyzed by flow cytometry. Only clones containing more than 90\% positive cells for the EGFP were further cultured and studied. These cells showed high and homogeneous alkaline phosphatase activity as well as uniform SSEA-1 immunostaining (data not shown). The selected Oct4-EGFP transfected cells were cultured in suspension, allowing EB formation, and displayed uniformly high fluorescence levels (Fig. 1C) after $48 \mathrm{~h}$. These EBs were used for further differentiation studies.

Differentiation of Oct4-EGFP transfected mouse ES cells. After LIF withdrawal, cells were cultured in suspension in order to generate EBs, which were maintained in culture for a maximum of $30 \mathrm{~d}$. Different studies were performed to evaluate the course of the differentiation process, including the analysis of green fluorescence and the expression level of genes related to pluripotency, as well as characteristic genes of the three primary germ layers. A representative experiment shown in Figure 2 demonstrates that green fluorescence, quantified by analysis of flow cytometry, decreased gradually in the EBs during their culture in absence of LIF. This result agreed with the parallel reduction in the Oct4 transcription level analyzed by RT-PCR (Fig. 2), in concordance with previous reports (Buehr et al., 2003). During the first $5 \mathrm{~d}$, the population of EGFP-positive cells was maintained over 95\%, without any significant decrease in the initial fluorescence intensity. However, there was an evident reduction of the EGFP intensity during the sixth day in culture, suggesting a synchronized decrease in the expression of Oct4. By day 8 of the EB culture, the population of EGFPpositive cells was dramatically reduced to $35 \%$ and the fluorescence gradually decreased onward. Interestingly, a slight fluorescence was still evident after $14 \mathrm{~d}$ that was increasing slowly during the last phase of culture reaching a $30 \%$ plateau. A significant increase in the Oct 4 expression was, in fact, observed after day 25 in culture. This increase was also corroborated by RT-PCR (Fig. 2). Parallel temporal analysis of differentiation markers was performed. The expression of $\alpha$-fetoprotein (AFP), used as a representative endoderm specific marker, as it is shown in Figure 2, gradually increased during the course of culture as an indicator of the ongoing differentiation processes. In parallel with AFP, there was a high level of beta-myosin heavy chain (MHC) expression after $14 \mathrm{~d}$, an established mesoderm marker. However, the expression of neurofilament 200 , analyzed as a neuroectoderm marker, was elevated since the first day, suggesting a neuroectodermal commitment of these cells in monolayer cultures (Roche et al., 2005) (Fig. 2). Additionally, we studied the expression of other markers associated with pluripotency, such as nanog or esgl (Cavaleri et al., 2003), by RT-PCR. Nanog expression evolved almost in parallel with Oct4, decreasing after $5 \mathrm{~d}$ of EB culture and increasing gradually after $25 \mathrm{~d}$. However, the expression of esg-1 was more accentuated, decreasing only slightly after $14 \mathrm{~d}$ (Fig. 2).

Analysis of VASA, DAZL, and Oct4 expression in residual undifferentiated cells. Oct4-EGFP transfected cells, which still showed fluorescence in the EBs after $20 \mathrm{~d}$ of culture, were isolated by cell sorting and further characterized. The presence of positive Oct4EGFP cells after a certain period of differentiation could possibly be caused by the presence of residual undifferentiated cells or be- 

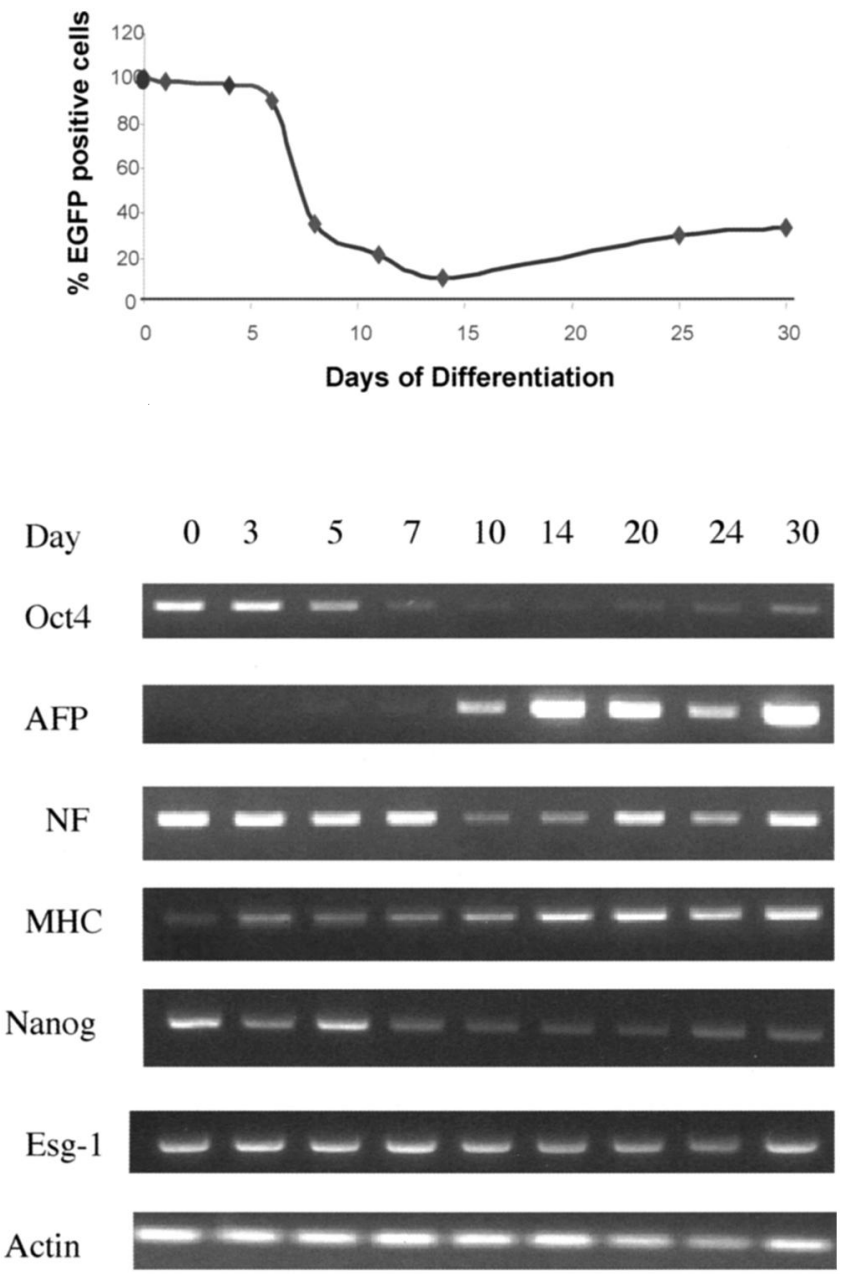

FIG. 2. Fluorescence decay in the embryoid bodies (EBs) from Oct4EGFP transfected cells at different days in culture and parallel expression of Oct4, $\alpha$-fetoprotein (AFP), neurofilament 200 (NF), beta-myosin heavy chain (MHC), Nanog, Esg-1, and actin, analyzed by reverse transcription/ polymerase chain reaction (RT-PCR). EGFP, enhanced green fluorescent protein.

cause of the possible generation of PGCs. In order to further analyze this question, we initially quantified the expression of VASA (Mvh), an established marker of PGCs (Tanaka et al., 2000), as well as Oct 4 by real-time PCR (Table 1). In EBs formed with parental transfected cells, the presence of VASA was negligible. However, in or-
TABLE 1

EXPRESSION OF VASA AND Oct4 BY REAL-TIME POLYMERASE CHAIN REACTION IN PARENTAL AND RESIDUAL Oct4-POSITIVE CELLS CULTURED IN MONOLAYER OR IN EMBRYOID BODIES (EBs) AS WELL AS THE EXPRESSION IN TESTIS

\begin{tabular}{lcc}
\hline \multicolumn{1}{c}{ Cell type or tissue } & VASA & Oct4 \\
\hline R1 parental transfected ES cells in & & \\
$\quad$ monolayer & $155 \pm 6$ & $8253 \pm 345$ \\
Rl parental transfected ES in EBs & $7 \pm 0.3$ & $72 \pm 3$ \\
Sorted residual cells in monolayer & $343 \pm 21$ & $4089 \pm 374$ \\
Sorted residual cells in EBs & $132 \pm 9$ & $1815 \pm 80$ \\
Testis & $7196 \pm 115$ & $6396 \pm 352$ \\
\hline
\end{tabular}

der to address this question, residual fluorescent Oct4-EGFP cells were sorted and expanded in the absence of LIF in monolayer for $15 \mathrm{~d}$ and then formed EBs for 14 additional days. Initially, we observed that the proliferation rate, during the first $72 \mathrm{~h}$ in culture of the residual fluorescent cell population, was similar to R1 ES cells, displaying doubling times of $10.12 \pm 0.75 \mathrm{~h}$ and $9.96 \pm 0.86 \mathrm{~h}$, respectively. The VASA expression of residual fluorescent cells was slightly higher than those observed in $\mathrm{Rl}$ undiffentiated transfected parental ES cells cultured in monolayer (Table 1), but they were considerably lower than the expected for PGCs growing in such conditions. The expression of VASA in residual cells was independent of the presence of LIF in the culture medium and decreased slightly after EB cultures (Table 1). In testis, which was used as a control, $7196 \pm 115$ normalized copies of VASA were detected (Table 1). Normalized copies of Oct4 detected in the fluorescent residual cells in monolayer cultures by real-time PCR were $50 \%$ lower than in parental ES cells (Table 1); this difference could be because residual cells were expanded in absence of LIF in order to maintain the conditions in which they were selected. Interestingly, after the EB culture period, residual fluorescent cells still displayed a significantly high number of copies of Oct 4 in comparison with parental Rl ES cells (Table 1). This is in agreement with the observed resistance of these residual cells to differentiate in standard conditions. Because of the slight increase of VASA expression displayed in the residual cells (Table 1), we additionally decided to analyze in parallel the expression of VASA and DAZL, another marker of PGCs (Lacham-Kaplan, 2004) by RT-PCR (Fig. 3). Both genes presented a small signal in undifferentiated ES cells, and they displayed a slight increase in sorted residual fluorescent cells after $14 \mathrm{~d}$ in culture. All these data suggest the possibility that the
FIG. 3. DAZL and VASA expression in D3 and Rl undifferentiated embryonic stem (ES) cells as well as in residual fluorescent cells analyzed by reverse transcription/polymerase chain reaction (RT-PCR). Testis (1); negative control (2); undifferentiated mouse D3 ES cells (3); R1-Oct4-GFP transfected undifferentiated cells (4); Rl transfected cells after 20 $\mathrm{d}$ in embryoid bodies (EBs) (5); sorted Oct4positive cells after $14 \mathrm{~d}$ in culture (6). Actin was used as a control.

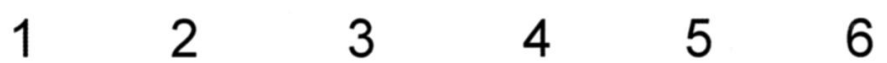

Dazl

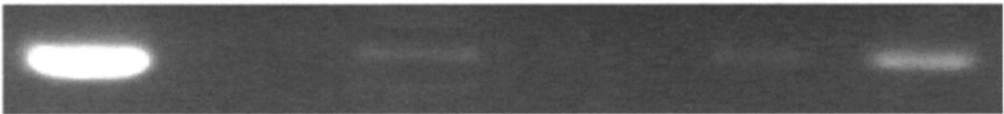

VASA

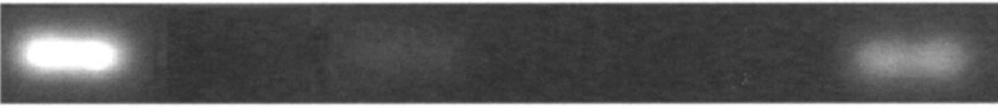

Actin

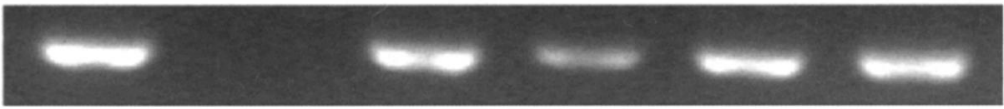



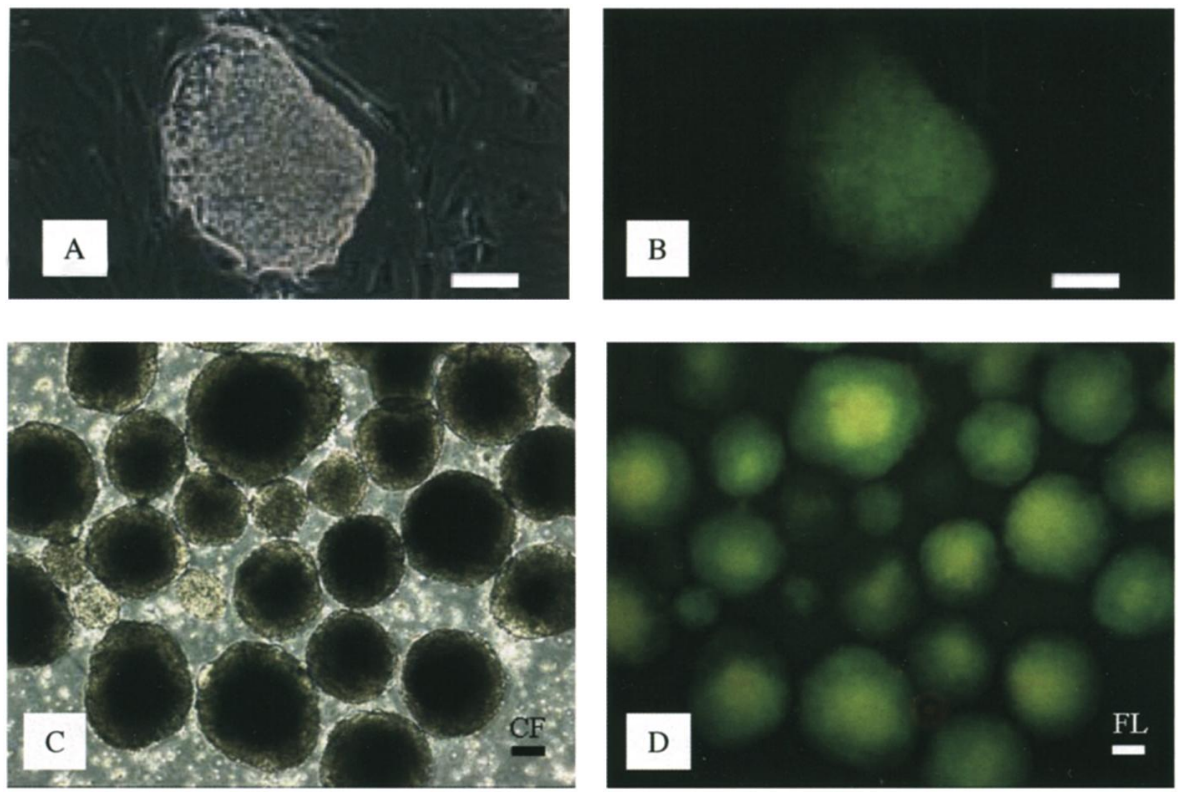

FIG. 4. Isolation and culture of residual fluorescent Oct4-EGFP transfected cells. $(A)$ Phase contrast and $(B)$ green fluorescence of residual Oct4-EGFP transfected cells sorted after $20 \mathrm{~d}$ in embryoid body (EB) culture and further expanded in monolayer for $15 \mathrm{~d}$ in absence of leukemia inhibitory factor (LIF) and without feeder layer. These cells were further cultured for 14 additional days forming EBs and visualized by $(C)$ phase contrast and $(D)$ green fluorescence. Bar, $100 \mu \mathrm{m}$. EGFP, enhanced green fluorescent protein.
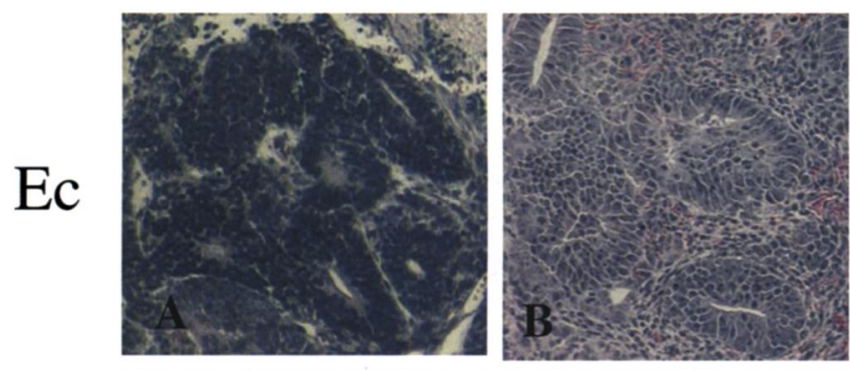

En
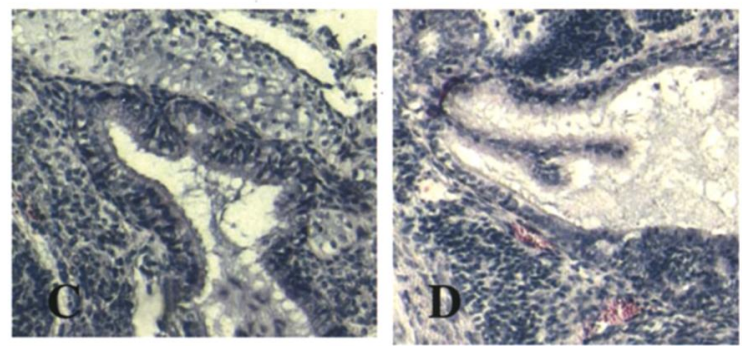

$\mathrm{Me}$
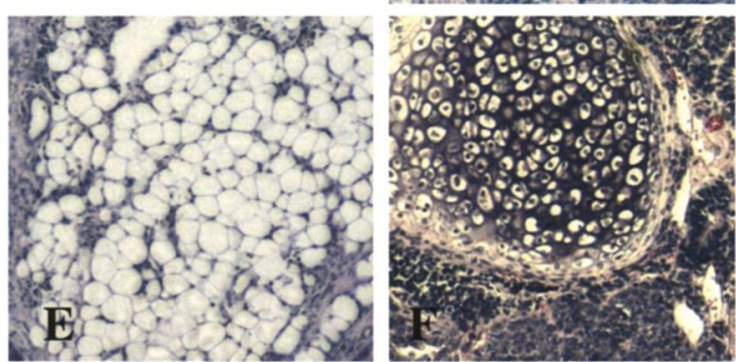

FIG. 5. Comparative histological analyses of tumors obtained from Rl embryonic stem (ES) cells (left, $A, C, D$ ) and fluorescent Oct4-EGFP residual cells expanded in absence of leukemia inhibitory factor (LIF) (right, $B, D$, $F)$. Paraffin-embedded sections were stained with hematoxylin and eosin. Sections through tumors contain a variety of tissue types derived of the three embryonic layers: ectoderm (Ec) neuroepithelium $(A, B)$; endoderm $(E n)$ ciliary epithelium $(C, D)$; mesoderm $(\mathrm{Me})$ adipocytes $(\mathrm{E})$ or condrocytes $(\mathrm{F})$. Magnification $\times 100(A, C, D, E, F), \times 200(B)$. EGFP, enhanced green fluorescent protein. population of undifferentiated residual cells could contain, additionally, a fraction of PGCs in an early stage of differentiation.

Monolayer cultures of fluorescent sorted cells growing in absence of LIF presented cell clusters with high and homogeneous fluorescence (Fig. $4 A$ and $B$ ), and some differentiated cells can be also observed in the outgrowth. The EBs generated with these cells displayed the characteristic core of necrosis, usually observed in these aggregates, which is caused by a limited flow of nutrients. However, in contrast to EBs derived from parental transfected ES cells that showed clear signals of differentiation, such as the beating activity or the expression of characteristic marker genes (Fig. 2), EBs from residual fluorescent cells did not show any evidence of differentiation, and they expressed high levels of fluorescence during the 14-d period they were in culture (Fig. $4 C$ and $D$ ). This result coincides with the significant expression of Oct4 in these EBs analyzed by real-time PCR (Table 1). Expression signals of AFP or MHC were not detected in these EBs by RT-PCR.

Tumorigenic potential of residual undifferentiated cells. We further studied the development of these residual undifferentiated cells after subcutaneous injection into immunodeficient mice. As a control, R1 pluripotent ES cells were also injected. After $30 \mathrm{~d}$, we observed the formation of teratomas in mice injected with R1 ES cells. Mice injected with $10^{6}$ residual fluorescent cells displayed a tumorigenic uniform mass after $90 \mathrm{~d}$. Apparently, the efficiency of residual cells inducing teratomas in terms of time was lower than R1 ES cells. If the number of cells injected were higher, tumors would have appeared earlier. In any case, tumors were found circumscribed, without apparent signs of invasiveness. The microscopic analysis of tumors from both types of cells showed a cellular diversity representative of the three different germ layers. Areas of neuroectoderm, ciliary epithelium, chondrocytes, or adipocytes are shown (Fig. 5). This result demonstrates that residual undifferentiated cells, in spite of evading differentiation in vitro, are capable of inducing teratomas with the same potency as Rl pluripotent ES cells. The Oct 4 expression in the tumors, originating from both R1 ES cells and residual fluorescent cells, was analyzed by semiquantitative 


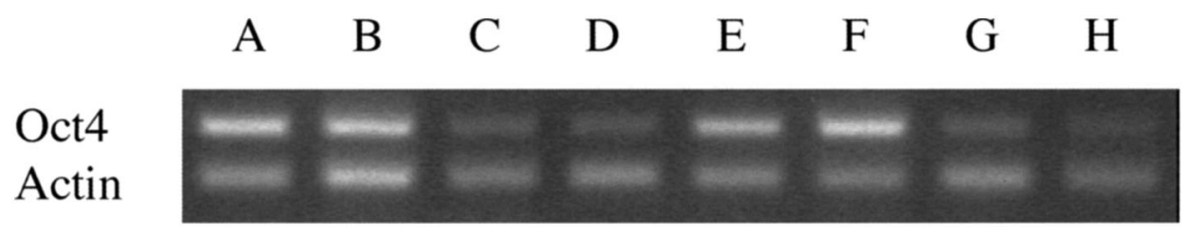

FIG. 6. Oct4 expression in undifferentiated embryonic stem (ES) cells and cells derived from tumors. The Oct4 expression was analyzed by semiquantitative polymerase chain reaction (PCR), normalizing densitometric values to the corresponding actin expression in different types of cells: Rl ES cells cultured in monolayer in presence of leukemia inhibitory factor (LIF) (A). Residual undifferentiated cells growing in monolayer in absence of LIF $(B)$. Tumoral tissue derived from Rl ES cells $(C)$ or derived from residual fluorescent cells $(D)$. Fluorescent Oct4+-positive cells derived from tumors and cultured in monolayer in absence of LIF without gelatin for $1 \mathrm{~d}(E)$ and $7 \mathrm{~d}$ $(F)$ or in the presence of gelatin for $7 \mathrm{~d}(G)$ or as embryoid bodies $(\mathrm{EBs})$ for $7 \mathrm{~d}(H)$.

FiG. 7. Phase contrast $(A)$ and green fluorescence $(B)$ of tumor-derived cells obtained after partial digestion of teratomas formed from fluorescent Oct4-EGFP residual cells expanded in monolayer in the absence of leukemia inhibitory factor (LIF). Bar, $100 \mu \mathrm{m}$. EGFP, enhanced green fluorescent protein.
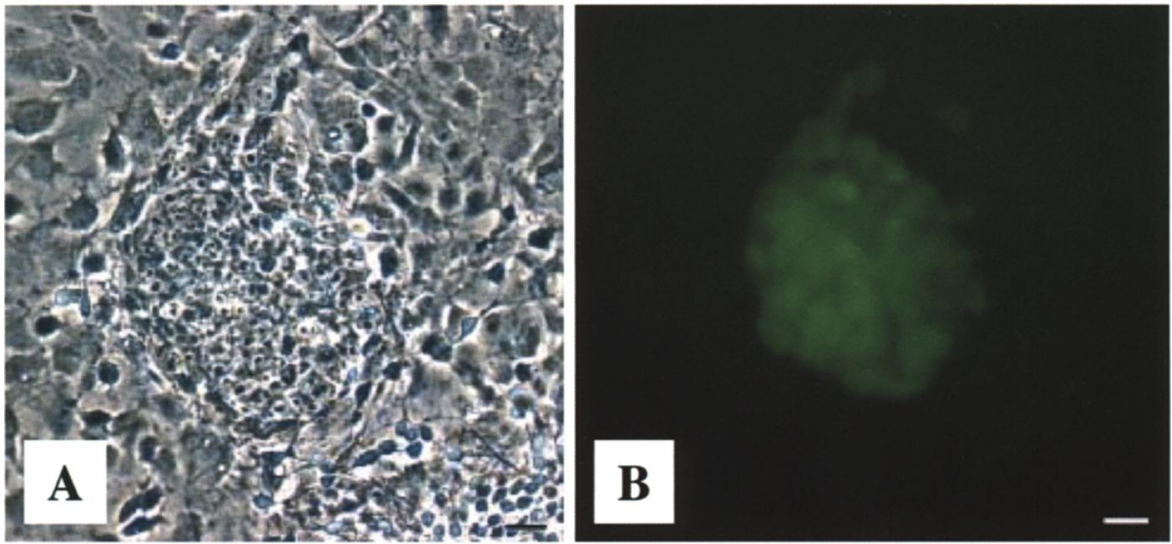

PCR, resulting in similar values in both cases, $0.5 \pm 0.05$ and 0.45 \pm 0.07 , respectively (Fig. 6). These values were lower than the obtained for the corresponding inoculated cells, according to the expected down-regulation that parallels differentiation during teratoma formation. This result demonstrated the presence of a similar fraction of undifferentiated cells in the teratomas derived from both types of cells. The presence of undifferentiated cells in the teratomas was supported by the selection and cloning of fluorescent cells from tumors developed after $90 \mathrm{~d}$ from residual positive Oct4-EGFP sorted cells (Fig. 7). The proliferation rate of these cells in the absence of LIF displayed doubling time similar to R1 ES cells. Additionally, these fluorescent cells, derived from tumors, could initiate in vitro differentiation processes with parallel Oct4 down-regulation, which can be visualized by the corresponding fluorescence decay in the outgrowth (Fig. 7) or by semiquantitative PCR. When they were cultured on gelatin dishes or forming EBs, Oct4 expression was down-regulated (Fig. $6 G$ and $H$ ), as opposed to the residual fluorescent cells.

Karyotype analysis. Chromosome number and karyotypes were analyzed in the various undifferentiated cell types used and characterized in the present study. Residual undifferentiated cells sorted from EBs after $20 \mathrm{~d}$ in culture displayed a high percentage of heteroploid metaphases, with more than $50 \%$ presenting two additional chromosomes (Fig. 8). In contrast, R1 ES cells showed a normal karyotype with a frequency over $75 \%$. It is known that the degree of heteroploidy can increase by passage in culture (Freshney, 2000). We used R1 or D3 mouse ES cell lines in passages 20 and 15, respectively, with similar results. Fluorescent cells, derived from teratomas, also showed evident karyotypical alterations (Fig. 8C). A detailed study using spectral karyotyping (Table 2) showed that R1 ES cells had, in fact, only occasional modifications. However, residual fluorescent cells significantly displayed karyotypical alterations, consisting mainly of an additional copy of chromosomes 8 and 9. This alteration was also observed in all studied metaphases from the fluorescent cells obtained from the corresponding teratomas. The presence of a high number of heteroploid metaphases in the residual cells isolated from EBs in vitro suggested the possibility that such chromosomal abnormalities could be the reason why these cells are selected in vitro, keeping possibly a nonregulated Oct4 expression. However, the corresponding Oct4 gene does not reside in either chromosome 8 or chromosome 9, which appear mainly in the karyotypical modifications observed in these residual cells. Furthermore, fluorescent cells from the derived teratomas, having the same chromosomal abnormalities, can differentiate in vitro. These results suggest that karyotypical alterations in residual undifferentiated cells do not derive directly from the original parental ES cells. Therefore, alterations probably occur during cell culture and can contribute, in some way, to select a cell subpopulation possessing intrinsic resistance to differentiate in vitro maintaining a prevalent undifferentiated phenotype; this restriction is overcome in an in vivo environment.

\section{DISCLSSION}

The mechanisms that regulate the differentiation of ES cells and extinction of pluripotency after blastocyst implantation during in vivo embryogenesis are not yet known. However, culture of mouse ES cells in vitro can allow the study of these processes in more 
A)

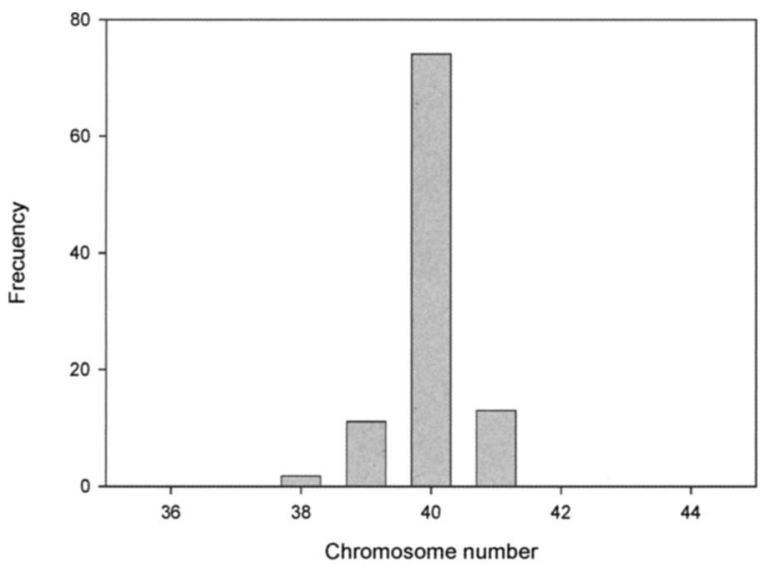

FIG. 8. Chromosome numbers of different undifferentiated Oct4-expressing cells used in the present study. (A) $\mathrm{Rl}$ mouse embryonic stem (ES) cells after passage 20. (B) Residual fluorescent Oct4-EGFP transfected cells sorted from 20-d embryoid body (EB) cultures. $(C)$ Fluorescent cells from teratomas originated with positive Oct4-EGFP residual cells. EGFP, enhanced green fluorescent protein.

B)

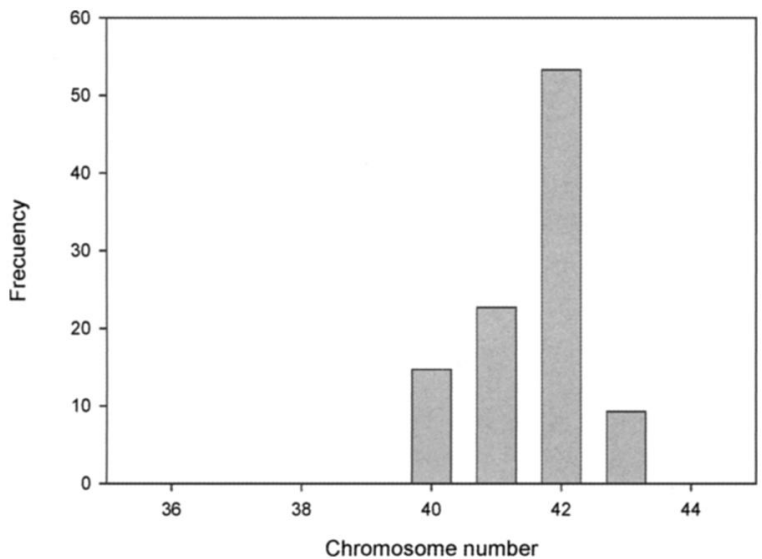

C)

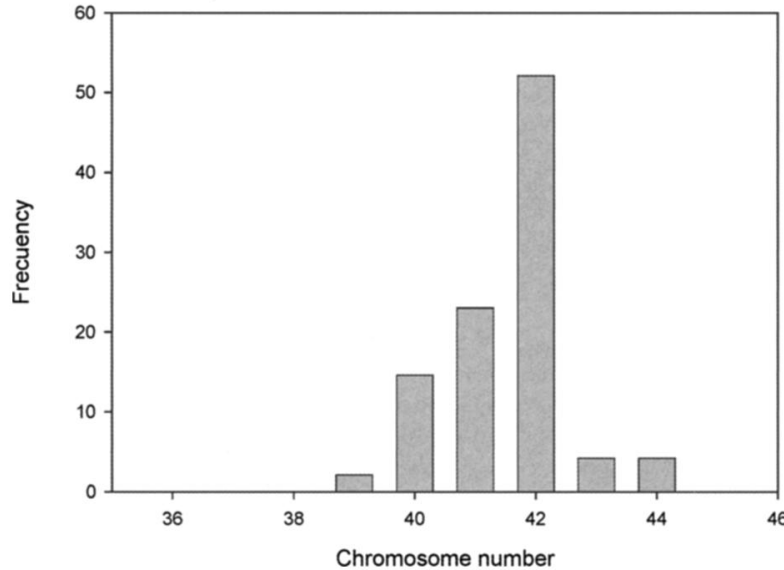


TABLE 2

SKY KARYOTYPE ANALYSIS OF DIFFERENT Oct4-EXPRESSING CELLS

\begin{tabular}{lll}
\hline \multicolumn{1}{c}{ Cell type } & No. cells $(\%)$ & \multicolumn{1}{c}{ Karyotype } \\
\hline $\begin{array}{l}\text { Rl mouse embryonic } \\
\text { stem cells }\end{array}$ & $8(80)$ & $40, \mathrm{XY}$ \\
& $1(10)$ & $40, \mathrm{XY} ; \mathrm{t}(4 ; 10)$ \\
& $1(10)$ & $1(10 \%)$ \\
Residual fluorescent & $6(60)$ & $42, \mathrm{XY} ;+8 ;+9$ \\
$\begin{array}{l}\text { Oct4-EGFP transfect- } \\
\text { ed cells }\end{array}$ & $1(10)$ & $43, \mathrm{XY} ;+8 ;+9 ;+10$ \\
& $1(10)$ & $43, \mathrm{XY} ;+2 ;+8 ;+11$ \\
& $1(10)$ & $43, \mathrm{XY} ; 8 ;+8 ;+9$ \\
Fluorescent cells from & $1(10)$ & $41, \mathrm{XY} ;+1 ;-5 ;-6 ;+8 ;+9$ \\
teratomas & $8(100)$ & $42, \mathrm{XY} ;+8 ;+9$ \\
\hline
\end{tabular}

EGFP $=$ enhanced green fluorescent protein.

detail. By using Oct4-EGFP R1 transfected cells, we have shown the presence of residual undifferentiated cells that remain in the EBs after standard culture conditions in the absence of LIF. The increase in Oct4-positive cells in an advanced culture stage can be partially caused by a greater proliferative potential of these cells compared with differentiated cells. Other cells that also express Oct4, such as PGCs, have been recently obtained from mouse ES cells, derived directly from blastocysts (Toyooka et al., 2003; Geijsen et al., 2004). We have demonstrated that the isolated Oct4positive residual cells expressed low levels of VASA by real-time PCR and that VASA expression did not rise during EB formation, suggesting that the possible presence of PGCs in the residual cell population should be very small. In any case, this result and the slight expression of VASA and DAZL, observed in parallel by RTPCR, also in residual cells, does not exclude the possibility that residual Oct4-positive cells could also contain a fraction of PGCs in an early stage of differentiation (Lacham-Kaplan, 2004). So far, the lack of specific markers that can clearly distinguish between undifferentiated ES cells and PGCs makes difficult any definitive conclusion in this respect. Further studies should be carried out in order to characterize with more detail the residual undifferentiated cells selected in vitro.

The presence of undifferentiated cells remaining in mouse ES cell cultures has been previously observed in monolayer cultures (Ying et al., 2003). The maintenance of Oct4 expression in this cell subpopulation was dependent on the presence of LIF (Viswanathan et al., 2003). For the first time, these undifferentiated residual cells from standard EB cultures have been studied and isolated in this investigation, demonstrating the presence of a LIF-independent population. A previous report established that the selective elimination of differentiated cells allowed the survival of undifferentiated cells in EBs, which maintained pluripotent capabilities, probably because of the absence of specific inductive signals that should be produced by the eliminated differentiated cells (Mountford et al., 1998). Additionally, this investigation has demonstrated that residual undifferentiated cells can be selected in EB cultures, even in the presence of differentiated cells. The residual cell subpopulation showed an undifferentiated phenotype in vitro, expressing high levels of Oct 4 and fluorescence, even after EBs formation. Interestingly, these cells were able to induce teratomas in nude mice, probably caused by specific signals in an in vivo environment that could reactivate some intracellular pathways. Restrictions of these resid- ual cells to follow differentiation processes in vitro in the EBs could be caused by the abnormal observed karyotype. The molecular mechanisms underlying the presence of these residual Oct4-expressing cells in vitro are being studied. Previous studies in which selected differentiated cells were obtained from transfected mouse ES cells expressing an antibiotic resistance in parallel did not show tumorigenic potential when implanted in mice (Soria et al., 2000; León-Quinto et al., 2004), probably because Oct4-positive residual cells could be eliminated by these specific selection protocols that use different antibiotics. The possibility that tumors could, in fact, appear in implanted mice after longer periods of study cannot be ruled out. When using other approaches, the selection of differentiated cells from mouse ES cells apparently cannot avoid these residual undifferentiated cells because tumor formation was reported after transplantation (Blyszczuk et al., 2003). The present study suggests that the control or the suppression of Oct4-expressing cells after specific in vitro protocols that leads to differentiated cells should be taken into account in order to assure further success in long-term grafts.

Improper regulation of Oct 4 or other genes related to a suitable differentiation response in the residual cells characterized in this study can be in fact the consequence of their in vitro behavior. The presence of an abnormal karyotype in these cells could be related with this effect. Recurrent karyotypic changes have also been recently described both in adult and in embryonic stem cell cultures of human origin (Draper et al., 2004; Rubio et al., 2005). The possible reactivation of the pluripotency showed by these residual cells in vivo in immunodeficient mice probably requires different conditions. Recently, the expression of Oct 4 has been reported in adult stem cells as well as tumor cells (Tai et al., 2005), suggesting the implication of this transcription factor in the promotion of carcinogenesis in certain circumstances. The signals and mechanisms that may condition the expression of this transcription factor, both in vivo and in vitro as well as in pathological conditions, should be further studied.

\section{ACKNOWLEDGMENTS}

We are grateful to Encarna Fuster for her technical assistance. In addition, we would like to express our appreciation to F. I. Aranda and M. Planelles for their expert assistance concerning cytological analysis of teratomas, to A. Campos for his support with FACS analysis, and to F. Galán and M. S. Aguilar for their assistance in the chromosome analysis. Finally, we would also like to thank Marie Rockstad and Jonathan Jones for constructive reading of the manuscript. This research was supported by the Generalitat Valenciana (GV 04B-659 and CTIDIB/2002/17), JAR (GV 04B-666) to E. R., and the Instituto Salud Carlos III (RCMN C03/08) to E. R.

\section{REFERENCES}

Blyszczuk, P.; Czyz, J.; Kania, G.; Wagner, M.; Roll, U.; St-Onge, L.; Wobus, A. M. Expression of Pax4 in embryonic stem cells promotes differentiation of nestin-positive progenitor and insulin-producing cells. Proc. Natl. Acad Sci. USA 100:998-1003; 2003.

Buehr, M.; Nichols, J.; Stenhouse, F.; et al. Rapid loss of oct-4 and pluripotency in cultured rodent blastocysts and derivative cell lines. Biol. Reprod. 68:222-229; 2003.

Cavaleri, F.; Scholer, H. R. Nanog: a new recruit to the embryonic stem cell orchestra. Cell 113:551-552; 2003.

Doetschman, T. C.; Einstetter, H.; Katz, M.; Schmidt, W.; Kemler, R. The in vitro development of blastocyst-derived embryonic stem cell lines: formation of visceral yolk sac, blood islands and myocardium. J. Embryol. Exp. Morphol. 87:27-45; 1985. 
Draper, J. S.; Smith, K.; Gokhale, P.; et al. Recurrent gain of chromosomes $17 \mathrm{q}$ and 12 in cultured human embryonic stem cells. Nat. Biotechnol. 22:53-54; 2004

Freshney, R. I. Culture of animal cells. In: Wiley-Liss; John Wiley \& Sons, New York, NY. 2000:229-257; 2000.

Gardner, R. L.; Rossant, J. Investigation of the fate of 4.5 day post-coitum mouse inner cell mass cells by blastocyst injection. J. Embryol. Exp. Morphol. 52:141-152; 1979.

Geijsen, N.; Horoschak, M.; Kim, K.; Gribnau, J.; Eggan, K.; Daley, G. Q. Derivation of embryonic germ cells and male gametes from embryonic stem cells. Nature 427:148-154; 2004.

Hopfl, G.; Gassmann, M.; Desbaillets, I. Differentiating embryonic stem cells into embryoid bodies. Methods Mol. Biol. 254:79-98; 2004.

Kim, J. H.; Auerbach, J. M.; Rodríguez-Gómez, J. A.; et al. Dopamine neurons derived from embryonic stem cells function in an animal model of Parkinson's disease. Nature 418:50-56; 2002.

Klug, M. G.; Soonpaa, M. H.; Koh, G. Y.; Field, L. J. Genetically selected cardiomyocytes from differentiating embryonic stem cells form stable intracardiac grafts. J. Clin. Invest. 98:216-224; 1996.

Lacham-Kaplan, O. In vivo and in vitro differentiation of male germ cells in the mouse. Reproduction 128:147-152; 2004.

León-Quinto, T.; Jones, J.; Skoudy, A.; Burcin, M.; Soria B. In vitro directed differentiation of mouse embryonic stem cells into insulin-producing cells. Diabetologia 47:1442-1451; 2004.

Mountford, P.; Nichols, J.; Zevnik, B.; O’Brien, C.; Smith, A. Maintenance of pluripotential embryonic stem cells by stem cell selection. Reprod. Fertil. Dev. 10:527-533; 1998.

Murray, P.; Edgar, D. The regulation of embryonic stem cell differentiation by leukaemia inhibitory factor (LIF). Differentiation 68:227-234; 2001.

Nagy, A.; Rossant, J.; Nagy, R.; Abramow-Newerly, W.; Roder, J. C. Derivation of completely cell culture-derived mice from early-passage embryonic stem cells. Proc. Natl. Acad. Sci. USA 90:8424-8428; 1993.

Nichols, J.; Zevnik, B.; Anastassiadis, K.; Niwa, H.; Klewe-Nebenius, D.; Chambers, I.; Schöler, H.; Smith, A. Formation of pluripotent stem cells in the mammalian embryo depends on the POU transcription factor Oct4. Cell 95:379-391; 1998.

Niwa, H. Molecular mechanism to maintain stem cell renewal of ES cells. Cell Struct. Funct. 26:137-148; 2001.

Niwa, H.; Miyazaki, J.; Smith, A. G. Quantitative expression of Oct-3/4 defines differentiation, dedifferentiation or self-renewal of ES. Nat. Genet. $24: 372-376 ; 2000$.
Prelle, K.; Vassiliev, I. M.; Vassilieva, S. G.; Wolf, E.; Wobus, A. M. Establishment of pluripotent cell lines from vertebrate species-present status and future prospects. Cells Tissues Organs 165:220-236; 1999.

Raz, R.; Lee, C. K.; Cannizzaro, L. A.; d'Eustachio, P.; Levy, D. E. Essential role of STAT3 for embryonic stem cell pluripotency. Proc. Natl. Acad. Sci. USA 96:2846-2851; 1999

Roche, E.; Burcin, M. M.; Esser, S.; Rudiger, M.; Soria, B. The use of gating technology in bioengineering insulin-secreting cells from embryonic stem cells. Cytotechnology 41:145-151; 2003.

Roche, E.; Sepulcre, P.; Reig, J. A.; Santana, A.; Soria, B. Ectodermal commitment of insulin-producing cells derived from mouse embryonic stem cells. FASEB J. 19:1341-1343; 2005.

Rosner, M. H.; Vigano, M. A.; Ozato, K.; Timmons, P. M.; Poirier, F.; Rigby, P.; Staudt, L. M. A POU-domain transcription factor in early stem cells and germ cells of the mammalian embryo. Nature 345:686-692; 1990.

Rubio, D.; García-Castro, J.; Martín, M. C.; de la Fuente, R.; Cigudosa, J. C.; Lloyd, A. C.; Bernad, A. Spontaneous human adult stem cell transformation. Cancer Res. 65:3035-3039; 2005.

Solter, D.; Knowles, B. B. Monoclonal antibody defining a stage-specific mouse embryonic antigen (SSEA-1) Proc. Natl. Acad. Sci. USA 75:5565-5569; 1978.

Soria, B.; Roche, E.; Berná, G.; León-Quinto, T.; Reig, J. A.; Martín, F. ESBioingineered insulin-secreting cells normalize glycemia in streptozotocin diabetic mice. Diabetes 49:157-162; 2000.

Tai, M. H.; Chang, C. C.; Olson, L. K.; Trosko, J. E. Oct4 expression in adult human stem cells: evidence in support of the stem cell theory of carcinogenesis. Carcinogenesis 26:495-502; 2005.

Tanaka, S. S.; Toyooka, Y.; Akasu, R.; Katoh-Fukui, Y.; Nakahara, Y.; Suzuki, R.; Yokoyama, M.; Noce, T. The mouse homolog of Drosophila Vasa is required for the development of male germ cells. Genes Dev. $14: 841-853 ; 2000$.

Thomson, J. A.; Itskovitz-Eldor, J.; Shapiro, S. S.; Waknitz, M. A.; Swiergiel, J. J.; Marshall, V. S.; Jones, J. M. Embryonic stem cell lines derived from human blastocysts. Science 282:1145-1147; 1998.

Toyooka, Y.; Tsunekawa, N.; Akasu, R.; Noce, T. Embryonic stem cells can form germ cells in vitro. Proc. Natl. Acad. Sci. USA 100:11457-11462; 2003.

Viswanathan, S.; Benatar, T.; Mileikovski, M.; Lauffenburger, D. A.; Nagy, A.; Zandstra, P. W. Supplementation-dependent differences in the rates of embryonic stem cell self-renewal, differentiation, and apoptosis. Biotechnol. Bioeng. 84:505-517; 2003.

Ying, Q. L.; Stavridis, M.; Griffiths, D.; Li, M.; Smith, A. Conversion of embryonic stem cells into neuroectodermal precursors in adherent monoculture. Nat. Biotechnol. 21:183-186; 2003. 